Article

\title{
Multi-Twin-SSB Modulation with Direct Detection Based on Kramers-Kronig Scheme for Long-Reach PON Downstream
}

\author{
Xiang Gao ${ }^{D}$, Yuancheng Cai, Bo Xu *, Xiaoling Zhang and Kun Qiu \\ School of Information and Communication Engineering, Key Laboratory of Optical Fiber Sensing and \\ Communications (Education Ministry of China), University of Electronic Science and Technology of China, \\ Chengdu 611731, China; gaoxiang9156@163.com (X.G.); uestccyc@163.com (Y.C.); \\ x.zhangmail@foxmail.com (X.Z.); kqiu@uestc.edu.cn (K.Q.) \\ * Correspondence: xubo@uestc.edu.cn
}

Received: 9 January 2019; Accepted: 18 February 2019; Published: 21 February 2019

\begin{abstract}
As the demand for high data volumes keeps increasing in optical access networks, transmission capacities and distance are becoming bottlenecks for passive optical networks (PONs). To solve this problem, a novel scheme based on multi-twin single sideband (SSB) modulation with direct detection is proposed and investigated in this paper. At the central office, two SSB signals are generated simultaneously with the same digital-to-analog converters (DACs). The twin-SSB signal is not only robust against frequency selected power fading introduced by chromatic dispersion (CD), but also improves the spectral efficiency (SE). By combining a twin-SSB technique with multi-band carrier-less amplitude/phase modulation (multi-CAP), different optical network units (ONUs) can be supported by flexible multi-band allocation based on software-reconfigurable optical transceivers. The Kramers-Kronig (KK) scheme is adopted on the ONU side to effectively mitigate the signal-signal beat interference (SSBI) induced by the square-law detection. The proposed system is extensively studied and validated with four sub-bands using $50 \mathrm{Gbps} 16$ quadrature amplitude modulation (QAM) modulation for each sub-band using numerical simulations. Digital pre-equalization is introduced at the transmitter-side to balance the performance of different ONUs. After system optimization, a bit error rate (BER) threshold for hard decision forward error correction (HD-FEC) code with $7 \%$ redundancy ratio $\left(\mathrm{BER}=3.8 \times 10^{-3}\right.$ ) can be reached for all ONUs over $50-\mathrm{km}$ standard single-mode fiber.
\end{abstract}

Keywords: fiber optics and optical communications; fiber optics links and subsystems; modulation

\section{Introduction}

Recently, emerging services such as $4 \mathrm{~K} / 8 \mathrm{~K}, 5 \mathrm{G}$ and virtual reality (VR)/ augmented reality (AR) have imposed challenging demands on passive optical networks (PONs) [1-6]. The future PONs should be able to provide on-demand modifications of traffic transmission policies through network re-configurability, flexibility and elasticity. For this purpose, software-defined networking (SDN) emerged as an effective technique to meet the demands [7]. When extending the SDN control to the physics layer of the network, it is able to offer highly desirable network functionalities. The effective use of SDN in PON systems showed its great potential in re-configurability, backward compatibility and elasticity [2].

In order to obtain higher throughput and larger capacity, researchers have tried different techniques for advanced modulation in PON transmissions, such as the quadrature amplitude modulation (QAM), pulse amplitude modulation (PAM) or the phase shift keying (PSK) based sub-carrier modulation (SCM) [8], the orthogonal frequency division multiplexing (OFDM) $[5,9]$, 
and the carrier-less amplitude/phase modulation (CAP) [2-4,10-12]. Among these different methods, multi-band CAP (multi-CAP) using intensity modulation and direct detection was proposed as a feasible solution for next generation PON due to its low cost and simple structure. By combining the dynamic software-reconfigurable embedded digital orthogonal filters in the digital signal processing (DSP) unit of optical transceivers, the functionality, upgradability and flexibility of the PON transmission link can be considerably enhanced through a centralized SDN controller. However, conventional CAP preferred double side band (DSB) modulation and the transmission distance were limited due to frequency selected power fading introduced by chromatic dispersion (CD) [3]. The transmission of single sideband (SSB) optical signal was considered to be more robust against fiber dispersion and power fading, but the signal-signal beat interference (SSBI) generated by square-law detection and aggravated by accumulated dispersion $[2,13-16]$ degrades the performance of the SSB system. Recently, different methods have been proposed to mitigate the SSBI effects. Refs. [2,13] introduced a large guard band between the optical carrier and the modulated signal, but this method will limit the achievable spectral efficiency (SE) significantly. For higher SE, DSP methods were applied to cancel the SSBI without guard bands. Ref. [14] used two-stage Hilbert superposition cancellation construction to cancel the 1st order and 2nd order interference. The transmission performance could be improved by this construction, but the computation complexity was intolerable. Ref. [15] proposed a novel digital receiver-based two-stage linearization filter for SSBI cancelling. The DSP structure of this filter allowed low computation complexity, but the method itself would introduce extra unwanted SSBI terms that limit the compensation gain. In 2016, a direct detection coherent receiver based on the Kramers-Kronig (KK) relations was proposed which showed predominant SSBI mitigation capability [16]. Under the minimum phase requirement, the KK-receiver could reconstruct an optical field from the detected amplitude waveform, which made it very similar to a coherent receiver. Compared with the SSBI mitigation schemes mentioned above, the KK-receiver can provide more effective SSBI mitigation. The reduction in computation complexity also indicates the scheme's great potential in low cost applications.

Another shortcoming of the SSB system is that a portion of the bandwidth of digital-to-analog converters (DACs) is wasted since it only carries one piece of sideband information. In order to make full utilization of DAC, a twin-SSB system was proposed $[13,17,18]$, in which two groups of independent signals were modulated onto the right-sideband (RSB) and the left-sideband (LSB), respectively. The twin-SSB application can almost double the SE for a direct detection system.

In this paper, we propose and investigate a novel scheme based on a combination of multi-CAP and twin-SSB techniques, which we call multi-twin-SSB, for long-reach high-speed PON downstream. The structure of multi-CAP allows the sub-bands to be dynamically scheduled via a centralized SDN-controller. A pair of Hilbert transform filters are utilized to generate the twin-SSB signal by making full use of the bandwidth of DACs so that the SE is almost doubled. Thanks to the SSB modulation, CD-induced power fading can be eliminated and longer transmission distance can supported for long-reach PON. At the optical network unit (ONU) side, KK-receiver is adopted to mitigate the SSBI effects from the square-law detection due to its predominant SSBI mitigation capability and low computation complexity. Furthermore, the downlink signal can be separated in the digital domain with cost-effective digitally filters as a conventional multi-CAP signal.

\section{Principle of Multi-Twin-SSB PON Downlink Transmission}

The main principle of the proposed multi-twin-SSB is combining the advantage of multi-CAP and twin-SSB modulation. Different sub-bands of the multi-CAP signal are supported by flexible and reconfigurable SDN-controller. The digital SSB signal is obtained in the transmitter-side DSP and then converted into an optical signal by an optical modulator. Only a single-end photodiode (PD) is needed on the receiver-side and the KK-receiver is applied to efficiently remove SSBI. The schematic diagram of the proposed multi-twin-SSB PON downlink transmission system is shown in Figure 1. In the central office of the optical line terminal (OLT), multiple wavelengths are used where each wavelength 
carries a multi-twin-SSB signal for multiple users. For flexible usage of the network resources, the parameters for digital orthogonal filters in the multi-CAP can be dynamically set for ONUs via the SDN-controller. The ONUs are divided into two groups with their signals modulated onto either the LSB or the RSB as demonstrated in the inset (II) (a) in Figure 1. Pre-equalization can be used to allocate different signal power to different ONUs if necessary. After the multi-CAP signals are generated for both sidebands, a pair of digital Hilbert transform filters are applied to obtain the two SSB signals with one located in the LSB and the other one in the RSB. The combined LSB and RSB signals are then converted to analog signals using a pair of DACs. An optical modulator is assumed to convert the electrical signal into an optical signal. For following KK processing on the receiver side, the twin-SSB signal should be transmitted together with large enough optical carrier power to meet the minimum phase requirement [16]. Multiple optical wavelengths carrying different multi-twin-SSB signals are multiplexed by a wavelength division multiplexing (WDM) multiplexer (MUX) to increase the transmission capacity. For the remote node (RN), the WDM signal is first de-multiplexed by a De-MUX; then, LSB and RSB signals are separated by a pair of optical band-pass filters (OBPFs) as shown in the insets (II) (c) and (d) in Figure 1.

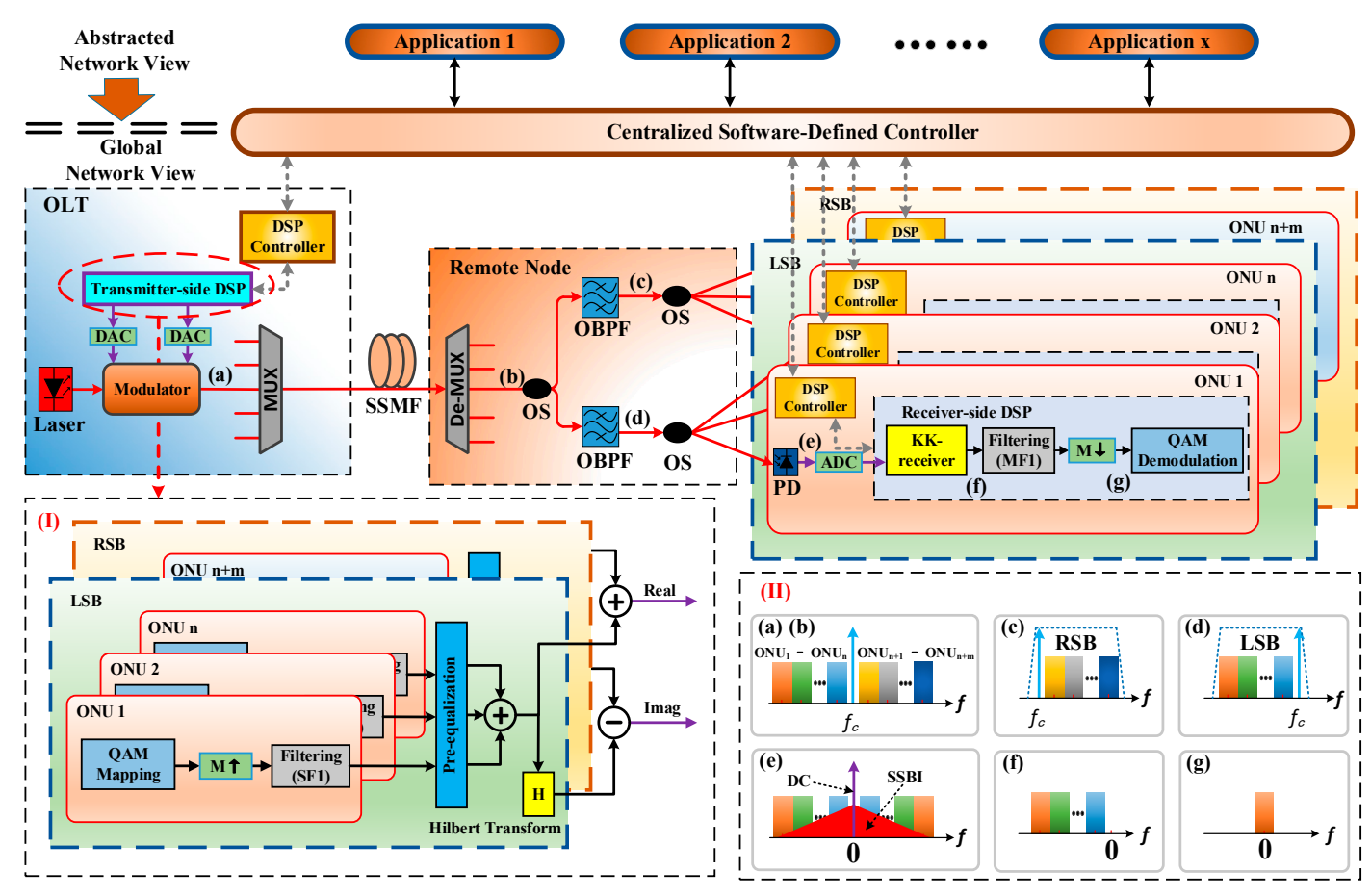

Figure 1. Schematic diagram of the proposed multi-twin single sideband (SSB) passive optical network (PON) downlink transmission system. Insets: (I) Transmitter-side digital signal processing (DSP) functions; (II) (a)-(g) represent signal spectra corresponding to points (a)-(g) marked in this proposed architecture which in detail (a): Multi-twin-SSB modulated signal on a single wavelength before multiplexer (MUX); (b): Multi-twin-SSB modulated signal on a single wavelength after De-MUX; (c): Right-sideband (RSB) signal after optical band-pass filter (OBPF); (d): Left-sideband (LSB) signal after OBPF; (e): LSB signal after direct detection for optical network unit (ONU) 1; (f): LSB after Kramers-Kronig (KK) receiver for ONU1; (g): Recovered signal for ONU1. SF, shaping filter; OS, optical splitter; MF, matched filter; DC, direct current. 
After the optical splitter, a single-end PD is used on each ONU side and the received electrical signal from the square-law operation of PD for the LSB signal can be described as [9]:

$$
\begin{aligned}
I(t) & =\left|A+\sum_{i=1}^{n} s_{i}(t)\right|^{2} \\
& =\underline{|A|^{2}}+\underline{2 A \sum_{i=1}^{n} \operatorname{Re}\left[s_{i}(t)\right]}+\underline{\sum_{i=1}^{n}\left|s_{i}(t)\right|^{2}}+\underline{\sum_{j=1, j \neq i}^{n} \sum_{i=1}^{n} s_{i}(t) s_{j}^{*}(t)}
\end{aligned}
$$

where $A$ is a constant from the optical carrier, $s_{i}(t)$ denotes the $i$-th ONU's SSB signal and $n$ is the number of ONUs in the LSB sideband. A similar expression exists for the received RSB signal. The superscript * denotes the complex conjugate and $\operatorname{Re}[x]$ stands for the real part of a complex number. The first term in Equation (1) is the DC component and the second term contains the desired signal. The third term is the SSBI of the desired ONU signal. The fourth term is the beat interference between different ONU signals. Note that the third and fourth terms are the PD-induced nonlinear distortions which occupy the same bandwidth as the signals. An illustration of the interference effect is shown in the inset (II) (e) of Figure 1 where the signals with spectrum closer to the DC component are expected to have more serious interference than the signals away from the DC. Effectively eliminating the nonlinear SSBI becomes crucial for successful signal separation and detection on the ONU side.

For the following signal processing after PD in the proposed scheme, Equation (1) is rewritten as:

$$
I(t)=\left|A+m_{S S B}(t)\right|^{2}
$$

where the sum of $s_{i}(t)$ is represented as a sum signal $m_{S S B}(t)$ which could be either LSB or RSB signal. If $|A|$ is large enough, which means that the minimum phase condition is satisfied, the KK scheme can be utilized in our scheme to reconstruct the SSB signal as [16]:

$$
\begin{aligned}
\phi(t) & =\frac{1}{\pi} p \cdot v \cdot \int_{-\infty}^{+\infty} d t^{\prime} \frac{\ln \left[\sqrt{I\left(t^{\prime}\right)}\right]}{t-t^{\prime}} \\
E_{m}(t) & =\left\{\sqrt{I(t)} \exp [ \pm j \phi(t)]-E_{0}\right\}
\end{aligned}
$$

where $\phi(t)$ is the phase part of the minimum phase signal and p.v. denotes the Cauchy principal value of the integral. $E_{m}(t)$ is the reconstructed complex SSB signal containing information from all sub-bands as illustrated in the inset (II) (f) of Figure 1. Note that ' + ' should be used for the RSB signal and '-' should be used for LSB signal in Equation (4). After that, the data of each user can be recovered using a matched filter (MF). Note that the MFs should also be dynamically set via the SDN-controller together with the corresponding shaping filters (SFs) on the OLT side.

\section{Results and Discussion}

In order to validate the proposed scheme, numerical simulations based on the Virtual Photonics Incorporated Transmission Maker (VPI TM) 9.0 (product of VPIphotonics company in Berlin of Germany) with co-simulations of Matrix Laboratory (MATLAB) were conducted. The schematic of the simulated system followed Figure 1 with some simplification. In the simulations, both the LSB and RSB were assumed to include two sub-bands making four ONUs in total. For each ONU, a pseudo random binary sequence (PRBS) with $2^{18}$ bits was used for QAM modulation and a root-raised-cosine (RRC) filter with a roll-off factor of $\beta=0.1$ was used as the shaping filter with a symbol rate of $R s=12.5$ Gbaud. It is well known that the roll-off factor is an important parameter for both system performance and SE [19]. In order to achieve a high SE for the PON system, a small roll-off factor of 0.1 was chosen in this work. In addition, 16-QAM modulation was used for CAP signal generating. Due to the non-ideal filtering effect of the OBPF to separate the LSB and RSB signals, a guard band 
of $G B$ was applied to reduce the residual crosstalk from the opposite sideband. Two sub-bands from LSB were used for ONU1 and ONU2 while the other two sub-bands from RSB for ONU3 and ONU4. The central frequencies of the sub-bands were then found to be $f_{1}=-G B-3 R s(1+\beta) / 2$, $f_{2}=-G B-R s(1+\beta) / 2, f_{3}=G B+R s(1+\beta) / 2$ and $f_{4}=G B+3 R s(1+\beta) / 2$, respectively. In the simulations, the sub-band signals in LSB or RSB satisfied the summed signal as in Equation (1).

For the cost consideration, a dual-drive Mach-Zehnder modulator (DDMZM) was used to generate the SSB signal simultaneously with the required optical carrier [17]. To achieve this, the real and imaginary parts of the twin-SSB signal were fed into the two arms of the DDMZM, which were biased at the quadrature point. The optical source used in the simulation had a working wavelength of $1550 \mathrm{~nm}$. The laser linewidth was assumed to be $1 \mathrm{MHz}$ for low-cost operation. Standard single-mode fiber (SSMF) with a length of 50-km was assumed for long-reach PON in this work. The attenuation coefficient of the SSMF was set at $0.2 \mathrm{~dB} / \mathrm{km}$. Other parameters of SSMF included dispersion coefficient $\mathrm{D}$ of $16 \mathrm{ps} / \mathrm{nm} / \mathrm{km}$, and nonlinear index of $2.6 \times 10^{-20} \mathrm{~m}^{2} / \mathrm{W}$. The optical signal to noise ratio (OSNR) was set to $35 \mathrm{~dB}$ by adding Gaussian white noise. The responsivity and dark current of the PD were set to $0.85 \mathrm{~A} / \mathrm{W}$ and $0.5 \mathrm{nA}$. After the received signal was obtained as in Equation (2) by a square-law operation, the summed SSB signal was reconstructed by the KK-receiver shown in Figure 1. For this purpose, the phase signal was firstly obtained using Equation (3) based on the received signal. Then, the complex signal was reconstructed by combining the recovered magnitude and phase of the signal as in Equation (4).

\subsection{Guard Band}

For twin-SSB signal recovery, the LSB and RSB signals need to be separated by a pair of OBPFs. To model its non-ideal nature in practice, the OBPF was modeled as a 4th order Gaussian filter with $3 \mathrm{~dB}$ bandwidth of $B w=2(1+\beta) R s+G B$ in this work. The bandwidth of the guard band is an important parameter for the system as it affects both the magnitude of sideband crosstalk and the spectrum efficiency. In order to find an optimum $G B$, Figure 2 shows the receiver's signal-to-noise ratio (SNR) variation for ONU1, ONU2, ONU3 and ONU4 as a function of guard band for the LSB and RSB signals in a B2B scenario.
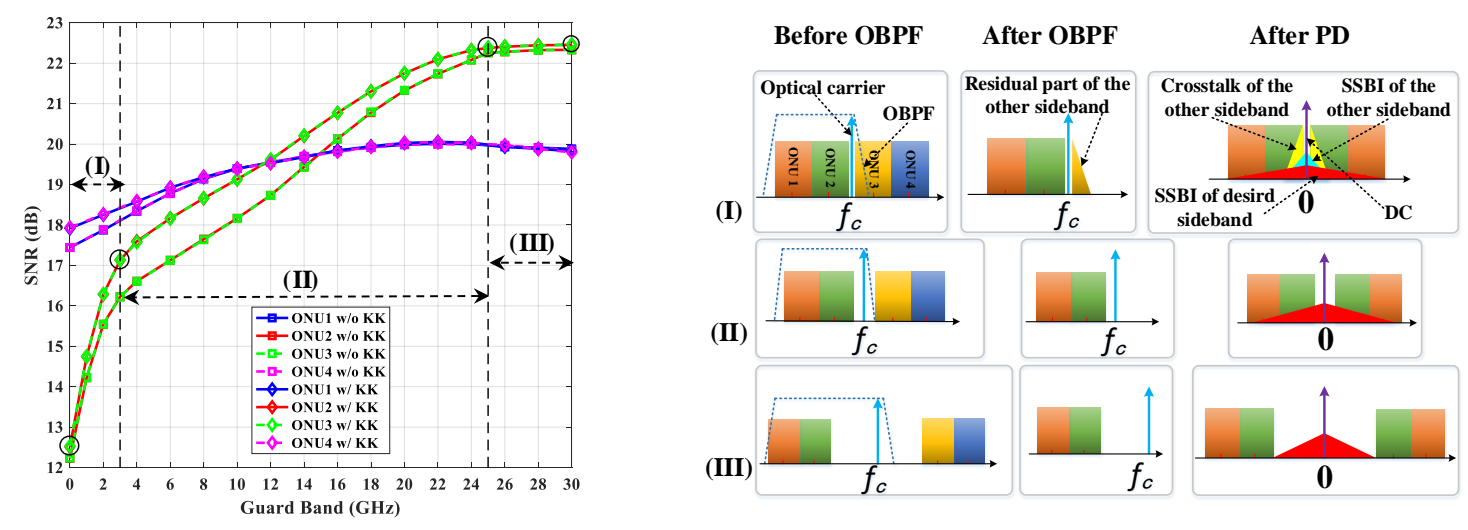

Figure 2. Signal-to-noise ratio (SNR) versus Guard Band for 12.5 Gbaud per sub-band 16 quadrature amplitude modulation (QAM) modulation in a back to back scenario. Insets: (I)-(III) represent the signal spectra before OBPF, after OBPF and after PD detection corresponding to stage (I)-(III) marked in the above curves figure.

As the performance of LSB sub-bands and RSB sub-bands were almost the same, we chose ONU1 and ONU2 (belonging to LSB) for the following discussion only. From Figure 2, it was found that the guard band parameter had much larger effects on the central sub-band for ONU2 than the outer sub-band for ONU1. For ONU2, the SNR changed with the guard band in three different stages as shown in the insets (I)-(III) in Figure 2. When a small GB of less than $3 \mathrm{GHz}$ was used for high spectrum 
efficiency, the SNR performance increased rapidly with increasing guard band as shown in stage (I) of Figure 2 because, in this stage, the SNR for the central sub-band was mainly dominated by the crosstalk from the other sideband due to non-ideal optical filtering. As the non-ideal filtering-induced crosstalk was very sensitive to the $G B$ parameter, a sharp SNR increasing was observed as the $G B$ increased in this stage. Due to the fact that KK receiver has no effect on this type of crosstalk, a close SNR performance was found for both cases without and with a KK receiver in stage (I), especially for small GB. However, if a $G B$ between $3 \mathrm{GHz}$ and $25 \mathrm{GHz}$ was selected as in stage (II) of Figure 2, the other sideband could be removed almost completely by the OBPF. In this case, the SNR performance of ONU2 was then dominated by the SSBI term from square-law detection and the KK-receiver was found to be able to improve about $1 \mathrm{~dB}$ SNR compared with the case without the KK-receiver. However, it was also observed that the rate of the SNR improvement got much slower in stage (II) compared to stage (I) as the GB increased. Moreover, the SNR performance of ONU2 eventually saturated when GB went beyond $25 \mathrm{GHz}$ as shown in stage (III) of Figure 2. In this stage, the SSBI term stayed at a different frequency location from the expected signals and could be efficiently removed by a digital filter. As a result, the KK-receiver showed no SNR improvement. Compared with ONU2, the outer sub-band for ONU1 showed much smaller SNR variation as GB varies. For ONU1, the KK-receiver showed no improvement compared to the conventional receiver except when $G B$ was very small because the SSBI products appear over a bandwidth equal to that of the original modulated SSB signal and the level of SSBI product appearing around a low frequency is much larger than that of high frequency. Furthermore, when $G B$ was lower than $13 \mathrm{GHZ}$, the outer sub-band for ONU1 only overlapped with the SSBI partially while the inner sub-band for ONU2 overlapped with the SSBI fully. As a result, ONU1 outperformed ONU2 in this case. When $G B$ was larger than $13 \mathrm{GHz}$, the SSBI would not overlap with the outer sub-band for ONU1 but its performance was still mainly limited by the filter edge roll off of the OBPF. Note that, in this case, the inner sub-band for ONU2 outperformed the outer sub-band for ONU1 because that ONU1 was located much closer to the edge of the OBPF. Based on the results shown in Figure 2, a $3 \mathrm{GHz}$ guard band was selected in our system for a good compromise between the system performance and spectrum efficiency.

\subsection{Optical Modulation Index}

As mentioned above, a DDMZM was used to generate the multi-twin-SSB signal together with the optical carrier required for a KK-receiver. However, the optical modulation index (OMI) which was related to the carrier to signal power ratio (CSPR) should be optimized carefully. The OMI was defined as $V_{r}^{r m s} / V_{\pi}$ [14], where $V_{r}^{r m s}$ was the root-mean-square of the electrical input signal to the DDMZM. Figure 3 shows the SNR performance of ONU1, ONU2, ONU3 and ONU4 as a function of OMI after 50-km transmission with a fiber launch power of $0 \mathrm{dBm}$.

A larger OMI is typically preferred for higher modulation efficiency. However, the MZM is known to have a nonlinear transfer function and a large OMI will introduce extra higher order nonlinear terms [9]. The nonlinear terms from the modulator deteriorated the SNR performance when OMI was higher than 0.08 as shown in Figure 3. On the other hand, a smaller OMI kept the modulator working under a linear range and a small SSB signal was generated with limited SNR. In this case, the system's SNR was determined by the channel noise and a sharp decreasing in SNR was found as OMI decreased from 0.08. About $0.9 \mathrm{~dB}$ and $2.3 \mathrm{~dB}$ improvements were observed with the KK-receiver for the ONU1 and ONU2, respectively, from Figure 3. The performances of ONU4 and ONU3 were almost the same as ONU1 and ONU2 because they were symmetric around the optical carrier. An optimum OMI value of 0.08 was obtained from Figure 3 , which would be used in the following simulations. 

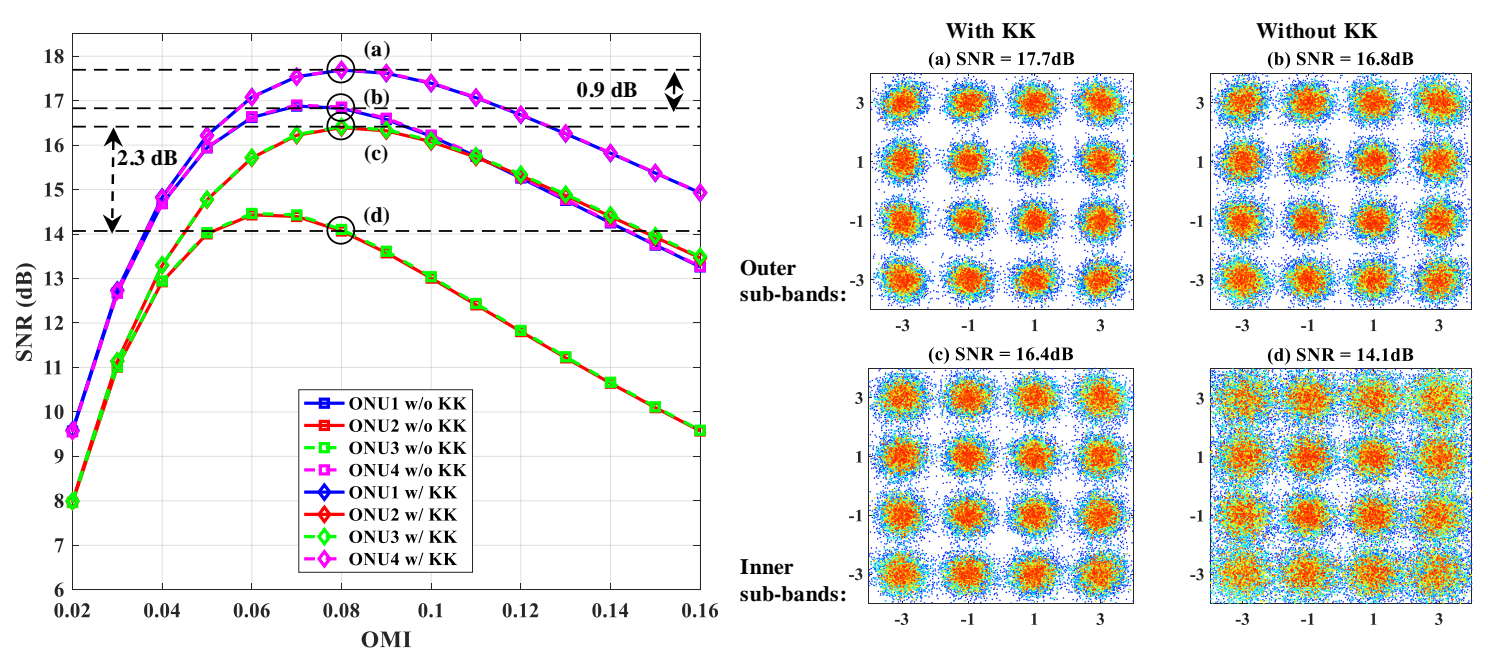

Figure 3. SNR versus OMI for 12.5 Gbaud per sub-band 16-QAM modulation after 50-km transmission. Insets: (a) and (b) represent the constellation diagrams for the outer sub-bands with and without KK-receiver at the optimum OMI, respectively; (c) and (d) represent the constellation diagrams for the inner sub-bands with and without a KK-receiver at the optimum optical modulation index (OMI) respectively.

\subsection{BER Performance}

Finally, the simulation results on the bit-error-rate (BER) performance of the multi-twin-SSB system with 16-QAM modulation after 50-km SSMF transmission with and without KK-receiver are presented in Figure 4a. In the simulations, the guard band was set to $3 \mathrm{GHz}$ and the OMI was 0.08. The two inner sub-bands for ONU2 and ONU3 were found to have worse BER performance than the two outer sub-bands for ONU1 and ONU4 since the ONU2 and ONU3 were closer to the optical carrier and the BERs were deteriorated mainly by the SSBI of the desired sideband. For example, ONU2 and ONU3 showed an error floor about $8.84 \times 10^{-3}$ for a conventional receiver without a KK-scheme. This high error floor made the system fail to work with the BER threshold of $3.8 \times 10^{-3}$ for the commonly-used hard-decision forward-error-correction (HD-FEC) code with 7\% redundancy ratio. However, if KK-receiver was available, the error floor could be reduced to $1.44 \times 10^{-3}$, which was lower than the 7\% redundancy ratio HD-FEC BER threshold. If the BER threshold of $2.4 \times 10^{-2}$ for a soft-decision FEC (SD-FEC) code with $20 \%$ redundancy ratio was used instead, the KK-receiver could provide about $1 \mathrm{~dB}$ received optical power (ROP) gain over the receiver without a KK-scheme for ONU2 and ONU3. Compared with ONU2 and ONU3, the two outer sub-bands for ONU1 and ONU4 showed better BER performance because the SSBI only overlapped the desired signals partially as discussed in Section 3.1. As a result, the BER floors of ONU1 and ONU4 with a KK-receiver and without a KK-receiver were about $2.55 \times 10^{-4}$ and $9.12 \times 10^{-4}$. About $0.7 \mathrm{~dB}$ ROP gain could be obtained for the KK-receiver at 7\% redundancy ratio HD-FEC BER threshold for ONU1 and ONU4.

From Figure 4a, it was also observed that the ONU2 and ONU3 had worse BER performance compared with the ONU1 and ONU4 because of the residual SSBI effect. In order to balance the performance between the ONUs, the power of different sub-band signals could be pre-equalized in the transmitter-side DSP. To do this, we increased the signal power of the two inner sub-bands while decreasing that for the two outer sub-bands. Figure $4 \mathrm{~b}$ shows the simulation results on BER performance after power pre-equalization. 

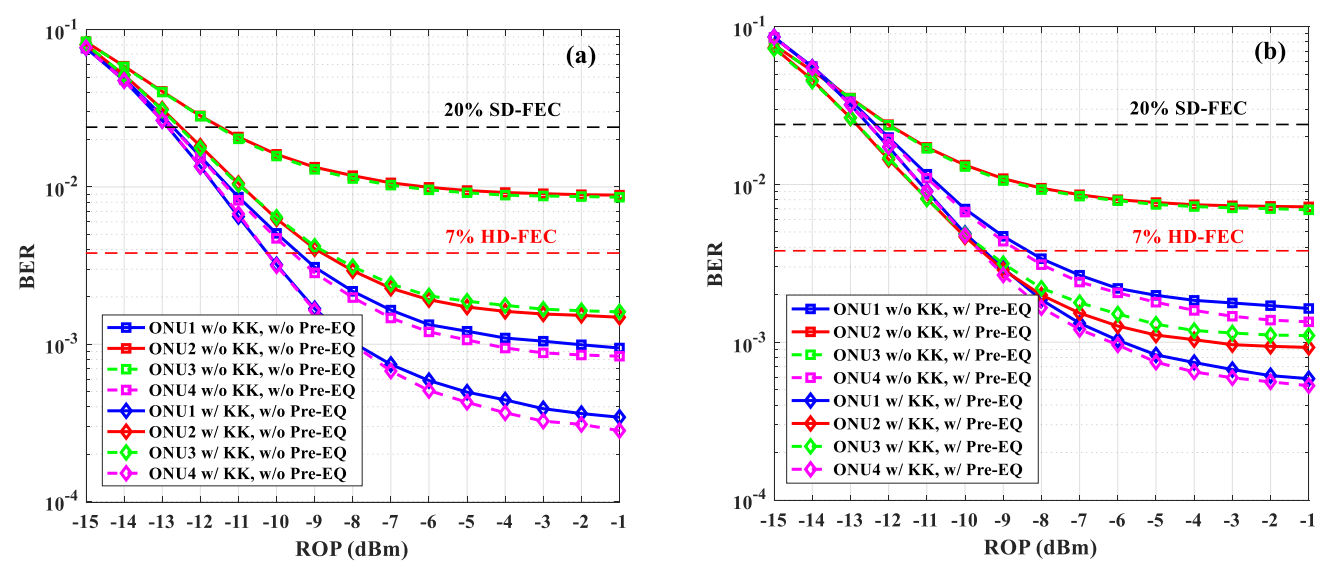

Figure 4. Bit-error-rate (BER) versus received optical power (ROP) for 12.5 Gbaud per sub-band 16-QAM modulation after 50-km transmission. (a): The BER performance without pre-equalization; (b): The BER performance with pre-equalization.

Comparing the results from Figure $4 \mathrm{~b}$ with Figure $4 \mathrm{a}$, the four ONUs were found to have a similar performance for the KK-receiver. For example, for the case without pre-equalization, if the $7 \%$ redundancy ratio HD-FEC BER threshold was used, the required ROP was about $-10.2 \mathrm{dBm}$ for ONU1 and ONU4, while a higher ROP of about $-8.8 \mathrm{dBm}$ was required for ONU2, ONU3. After pre-equalization, the required ROP became $-9.5 \mathrm{dBm}$ for all ONUs. Note that the pre-equalization could be dynamically controlled via the centralized SDN controller and easily implemented in the DSP-based transmitters.

\section{Conclusions}

In conclusion, a novel solution based on a multi-twin-SSB technique for long-reach high-speed PON downstream was proposed and investigated in this paper. Multi-twin-SSB combined the advantages of multi-CAP and twin-SSB modulation. Different ONUs could be supported by flexible multi-band allocation based on the software-reconfigurable optical transceivers. Compared with the conventional multi-CAP system, the employment of the twin-SSB technique generated two SSB signals without increasing the DAC bandwidth requirements. The CD-induced power fading could also be eliminated and the transmission distance could be improved. A single DDMZM was applied to modulate the twin-SSB signal on the transmitter-side while a low-cost single-end PD was used on the receiver-side. To efficiently remove the SSBI from the square-law detection, the KK-receiver was adopted with a careful optimization on the guard band between the two sidebands and the OMI of the DDMZM. To validate the proposed scheme, numerical simulations with four ONUs using $50 \mathrm{Gbps}$ 16-QAM modulation for each ONU was conducted for transmission over 50-km SSMF. Successful separation and receiving of each sub-band from the twin-SSB signal was achieved, which was able to almost double the transmission capacity. By the application of KK-receiver, the BER performance was improved by almost an order of magnitudes for the central sub-bands near the optical carrier. Digital pre-equalization was used on the transmitter-side DSP to balance the performance between the sub-bands. After pre-equalization, the required ROP was about $-9.5 \mathrm{dBm}$ for all sub-bands to reach the BER threshold for the commonly-used HD-FEC code with a $7 \%$ redundancy ratio. We believe that the multi-twin-SSB technique with direct detection based on a KK-receiver will be a simple and low-cost solution for long-reach high-speed PON downstream in the future.

Author Contributions: Conceptualization, X.G. and X.Z.; methodology, X.G. and Y.C.; software, X.G.; validation, B.X. and K.Q.; formal analysis, B.X.; investigation, X.G.; resources, X.G. and B.X.; data curation, X.G. and Y.C.; writing - original draft preparation, X.G. writing_-review and editing, Y.C., B.X., and X.Z.; visualization, X.G. and X.Z.; supervision, B.X. and K.Q.; project administration, B.X. and K.Q.; funding acquisition, B.X. and K.Q.

Funding: This research received no external funding. 
Acknowledgments: This work is supported in part by National Natural Science Foundation of China NSFC (No. 61420106011, No. 61871082 and No. 61471088) and the National High Technology Research and Development Program of China (863 Program) (2015AA015501).

Conflicts of Interest: The authors declare no conflict of interest.

\section{References}

1. Guo, Y.; Yin, Y.; Song, Y.; Huang, M.; Li, Y.; Kuang, G.; Fu, Z.; Huang, X.; Cai, P.; Ma, Z.; et al. Demonstration of 25Gbit/s per Channel NRZ Transmission with $35 \mathrm{~dB}$ Power Budget using 25G Ge/Si APD for Next Generation 100G-PON. In Proceedings of the Optical Fiber Communication Conference, Los Angeles, CA, USA, 19-23 March 2017.

2. Zhang, X.; Zhang, C.; Chen, C.; Jin, W.; Qiu, K. All-optical VPN utilizing DSP-based digital orthogonal filters access for PONs. Opt. Commun. 2018, 413, 347-352. [CrossRef]

3. Zhang, J.; Yu, J.; Li, F.; Chi, N.; Dong, Z.; Li, X. $11 \times 5 \times 9.3 \mathrm{~Gb} / \mathrm{s}$ WDM-CAP-PON based on optical single-side band multi-level multi-band carrier-less amplitude and phase modulation with direct detection. Opt. Express 2013, 21, 18842-18848. [CrossRef] [PubMed]

4. Wei, J.; Giacoumidis, E. Multi-band CAP for Next-Generation Optical Access Networks Using 10-G Optics. J. Lightwave Technol. 2018, 36, 551-559. [CrossRef]

5. He, H.; Li, J.; Bi, M.; Hu, W. 20-Gbps low cost WDM-OFDM-PON downstream transmission with tunable filter and linear APD module. Chin. Opt. Lett. 2014, 12, 040603-040607. [CrossRef]

6. Gebrewold, S.; Bonjour, R.; Barbet, S.; Maho, A.; Brenot, R.; Chanclou, P.; Brunero, M.; Marazzi, L.; Parolari, P.; Totovic, A.; et al. Self-Seeded RSOA-Fiber Cavity Lasers vs. ASE Spectrum-Sliced or Externally Seeded Transmitters-A Comparative Study. Appl. Sci. 2015, 5, 1922-1941. [CrossRef]

7. Bolea, M.; Giddings, R.P.; Bouich, M.; Aupetit-Berthelemot, C.; Tang, J.M. Digital Filter Multiple Access PONs With DSP-Enabled Software Reconfigurability. J. Opt. Commun. Netw. 2015, 7, 215-222. [CrossRef]

8. Gutiérrez, F.; Martin, E.; Perry, P.; Ellis, A.; Barry, L. Calculation of Receiver Sensitivities in (Orthogonal) Subcarrier Multiplexing Microwave-Optical Links. Appl. Sci. 2017, 7, 184. [CrossRef]

9. Peng, W.-R.; Wu, X.; Arbab, V.R.; Feng, K.-M.; Shamee, B.; Christen, L.C.; Yang, J.-Y.; Willner, A.; Chi, S. Theoretical and Experimental Investigations of Direct-Detected RF-Tone-Assisted Optical OFDM Systems. J. Lightwave Technol. 2009, 27, 1332-1339. [CrossRef]

10. Chi, N.; Zhao, J.; Wang, Z. Bandwidth-efficient visible light communication system based on faster-than-Nyquist pre-coded CAP modulation. Chin. Opt. Lett. 2017, 15, 080601.

11. Wang, Z.; Tao, L.; Wang, Y.; Chi, N. 56 Gb/s direct-detection polarization multiplexing multi-band CAP transmission. Chin. Opt. Lett. 2015, 13, 080602-080605. [CrossRef]

12. Iglesias Olmedo, M.; Tianjian, Z.; Bevensee Jensen, J.; Qiwen, Z.; Xu, X.; Monroy, I.T. Towards 400GBASE 4-lane Solution Using Direct Detection of MultiCAP Signal in $14 \mathrm{GHz}$ Bandwidth per Lane. In Proceedings of the Optical Fiber Communication Conference/National Fiber Optic Engineers Conference 2013, Anaheim, CA, USA, 17-21 March 2013.

13. Chen, M.; Peng, M.; Zhou, H.; Zheng, Z.; Tang, X.; Maivan, L. Receiver sensitivity improvement in spectrally-efficient guard-band twin-SSB-OFDM using an optical IQ modulator. Opt. Commun. 2017, 405, 259-264. [CrossRef]

14. Zhu, M.; Zhang, J.; Yi, X.; Song, Y.; Xu, B.; Li, X.; Du, X.; Qiu, K. Hilbert superposition and modified signal-to-signal beating interference cancellation for single side-band optical NPAM-4 direct-detection system. Opt. Express 2017, 25, 12622-12631. [CrossRef] [PubMed]

15. Li, Z.; Sezer Erkilinc, M.; Maher, R.; Galdino, L.; Shi, K.; Thomsen, B.C.; Bayvel, P.; Killey, R.I. Two-Stage Linearization Filter for Direct-Detection Subcarrier Modulation. IEEE Photonics Technol. Lett. 2016, 28, 2838-2841. [CrossRef]

16. Mecozzi, A.; Antonelli, C.; Shtaif, M. Kramers-Kronig coherent receiver. Optica 2016, 3, 1220. [CrossRef]

17. Zhang, L.; Zuo, T.; Mao, Y.; Zhang, Q.; Zhou, E.; Liu, G.N.; Xu, X. Beyond 100-Gb/s Transmission Over 80-km SMF Using Direct-Detection SSB-DMT at C-Band. J. Lightwave Technol. 2016, 34, 723-729. [CrossRef]

18. Zhu, Y.; Ruan, X.; Chen, Z.; Jiang, M.; Zou, K.; Li, C.; Zhang, F. $4 \times 200$ Gb/s Twin-SSB Nyquist Subcarrier Modulation WDM Transmission over 160km SSMF with Direct Detection. In Proceedings of the Optical Fiber Communication Conference, Los Angeles, CA, USA, 19-23 March 2017. 
19. Harako, K.; Suzuki, D.; Hirooka, T.; Nakazawa, M. Roll-off factor dependence of Nyquist pulse transmission. Opt. Express 2016, 24, 21986-21994. [CrossRef] (CC BY) license (http://creativecommons.org/licenses/by/4.0/). 